\title{
A CLASS OF AXIALLY SYMMETRIC STATIONARY EXACT SOLUTIONS OF EINSTEIN'S VACUUM FIELD EQUATIONS
}

\author{
M. D. PATEL
}

(Received 1 February 1978)

\begin{abstract}
Einstein's vacuum field equations of an axially symmetric stationary rotating source are studied. Using the oblate spheroidal coordinate system, a class of asymptotically flat solutions representing the exterior gravitational field of a stationary rotating oblate spheroidal source is obtained. Also it is proved that an analytic axisymmetric and stationary distribution of dust cannot be the source for the gravitational field described by the axisymmetric stationary metric.
\end{abstract}

\section{Introduction}

The study of exact solutions of the general relativity field equations for empty space-time is of some interest to physicists because these solutions are felt to correspond to the gravitational fields external to matter distributions which are in some sense localized. The exact solutions of Einstein's vacuum field equations with time symmetry and cylindrical symmetry have been obtained by several authors, in particular, Rosen [8], Bonnor [1], Weber and Wheeler [10] and Marder [4]. An infinite cylinder with finite cross-section is the source of the gravitational fields in these solutions. In astrophysics the sources of gravitation are rotating axially symmetric finite bodies and hence the solutions of Einstein's field equations corresponding to the axially symmetric, rotating stationary source may be more suitable to interpret real physical situations. Papapetrou [6] has given a class of exact, axially symmetric stationary solutions of Einstein's field equations for the empty space-time. Kerr [3] has presented the best known solution representing external fields for rotating matter distributions. The equilibrium shape of a rotating star is an oblate spheroid. Misra [5] used oblate spheroidal coordinates to obtain static solutions of the empty space-time field equations. Patel [7] has also used this 
coordinate system in obtaining the axially symmetric zero mass meson solutions of Einstein's equations. In this paper oblate spheroidal coordinates are again used to obtain a class of asymptotically flat solutions of the vacuum field equations.

\section{The coordinate system and vacuum field equations}

To discuss the gravitational fields of a stationary, rotating star, the best line element is the axisymmetric stationary space-time metric given by Chandrasekhar and Friedman [2]

$$
d s^{2}=-e^{2 \delta} d t^{2}+e^{2 \sigma}(d \phi-w d t)^{2}+e^{2 \beta}\left(d r^{2}+d z^{2}\right),
$$

where $\beta, \delta, \sigma$ and $w$ are functions of $r$ and $z$ only. The boundary of a rotating star is a surface of revolution and, under balance of self gravitation and the rotational effect, the star becomes an oblate spheroid. Hence it will be more suitable to use oblate spheroidal coordinates.

The oblate spheroidal coordinates $(\phi, \theta, \alpha)$ are defined by

$$
\left.r=a \sqrt{[}\left(1+\theta^{2}\right)\left(1-\alpha^{2}\right)\right] ; \quad z=a \theta \alpha ; \quad \phi=\phi,
$$

where $r, z, \phi$ are the usual cylindrical polar coordinates. Here $\theta$ ranges from 0 to $\infty$ and $\alpha$ from -1 to +1 . The surface $\theta=0$ is a disc of radius $a$ in the $x y$-plane. The surface $\alpha=0$ is the $x y$-plane except the part inside a circle of radius $a$ centred at the origin. The surfaces $\theta=$ constant $>0$ are flattened spheroids of thickness $2 a \theta$ through the axis of symmetry and are of the radius $a \sqrt{ }\left(1+\theta^{2}\right)$ in the equatorial plane. The surface $\alpha=1$ is the positive $z$-axis and the surface $\alpha=-1$ is the negative $z$-axis. The surfaces $\alpha=$ constant are hyperboloids of one sheet, asymptotic to the cone of semivertical angle $\cos ^{-1} \alpha$ and with $z$-axis as the axis of the cone. We will number the coordinates as $x^{0}=t, x^{1}=\phi, x^{2}=\theta$ and $x^{3}=\alpha$.

Now, under the transformation (2.2), the metric (2.1) becomes

$$
d s^{2}=-e^{2 \delta} d t^{2}+e^{2 \sigma}(d \phi-w d t)^{2}+a^{2}\left(\theta^{2}+\alpha^{2}\right) e^{2 \beta}\left(\frac{d \theta^{2}}{1+\theta^{2}}+\frac{d \alpha^{2}}{1-\alpha^{2}}\right),
$$

where $\beta, \sigma, \delta$ and $w$ are functions of $\theta$ and $\alpha$ only. The non-vanishing components of the Einstein tensor for the metric (2.3) are

$$
\begin{gathered}
G_{0}^{0}=\frac{1}{2} A\left[\Delta(\delta-\sigma)-\left(2+\theta^{2}-\alpha^{2}\right) \Delta \beta+\left(\theta^{2}+\alpha^{2}\right)\left(1+\theta^{2}\right)(\delta+\sigma)_{2} \beta_{2}\right. \\
-\left(1-\alpha^{2}\right)(\delta+\sigma)_{3}\left(\theta^{2}+\alpha^{2}\right) \beta_{3}-\left(1+\theta^{2}\right)^{2}\left\{\delta_{2}^{2}+\sigma_{2}^{2}+(\delta+\sigma)_{22}\right\} \\
\left.-\left(1-\alpha^{2}\right)^{2}\left\{\delta_{3}^{2}+\sigma_{3}^{2}+(\delta+\sigma)_{33}\right\}\right] \\
-1 A\left[\left(1-\theta^{4}\right) w_{2}^{2}+\left(1-\alpha^{4}\right) w_{3}^{2}+2 w \Delta w+2 w\left(1+\theta^{2}\right) w_{2}(3 \sigma-\delta)_{2}\right. \\
\left.+2 w\left(1-\alpha^{2}\right) w_{3}(3 \sigma-\delta)_{3}\right] \exp (2 \sigma-2 \delta),
\end{gathered}
$$




$$
\begin{aligned}
& G_{1}^{1}=\frac{1}{2} A\left[\Delta(\sigma-\delta)-\left(2+\theta^{2}-\alpha^{2}\right) \Delta \beta+2\left(1+\theta^{2}\right)(\delta+\sigma)_{2}(\sigma-\delta)_{2}\right. \\
& +2\left(1-\alpha^{2}\right)(\delta+\sigma)_{3}(\sigma-\delta)_{3}+\left(1+\theta^{2}\right)^{2}\left\{\delta_{2}^{2}+\sigma_{2}^{2}+(\delta+\sigma)_{22}\right\} \\
& +\left(\theta^{2}+\alpha^{2}\right)\left\{\left(1+\theta^{2}\right) \beta_{2}(\delta+\sigma)_{2}-\left(1-\alpha^{2}\right) \beta_{3}(\delta+\sigma)_{3}\right\} \\
& \left.-\left(1-\alpha^{2}\right)^{2}\left\{\delta_{3}^{2}+\sigma_{3}^{2}+(\delta+\sigma)_{33}\right\}\right] \\
& +\frac{1}{4} A\left[2 w\left\{\Delta w+\left(1+\theta^{2}\right) w_{2}(3 \sigma-\delta)_{2}+\left(1-\alpha^{2}\right) w_{3}(3 \sigma-\delta)_{3}\right\}\right. \\
& \left.+\left(1+\theta^{2}\right)\left(3+\theta^{2}\right) w_{2}^{2}+\left(1-\alpha^{2}\right)\left(3-\alpha^{2}\right) w_{3}^{2}\right] \exp (2 \sigma-2 \delta) \text {. } \\
& G_{2}^{2}=\frac{1}{2} A\left[\left(\theta^{2}+\alpha^{2}\right) \Delta \beta-\Delta(\delta+\sigma)+\frac{2\left(1+\theta^{2}\right)\left(1-\alpha^{2}\right)}{\theta^{2}+\alpha^{2}}\left\{\alpha(\delta+\sigma)_{3}-\theta(\delta+\sigma)_{2}\right\}\right. \\
& +\left(1+\theta^{2}\right)^{2}\left\{\sigma_{2}^{2}+\delta_{2}^{2}+(\sigma+\delta)_{22}-2 \beta_{2}(\sigma+\delta)_{2}\right\} \\
& -\left(1-\alpha^{2}\right)^{2}\left\{\sigma_{3}^{2}+\delta_{3}^{2}+(\sigma+\delta)_{33}\right\}+\left(1-\alpha^{2}\right)\left(2+\theta^{2}-\alpha^{2}\right) \beta_{3}(\delta+\sigma)_{3} \\
& +\left(\theta^{2}+\alpha^{2}\right)\left(1+\theta^{2}\right) \beta_{2}(\delta+\sigma)_{2}-2\left(1+\theta^{2}\right)(\delta+\sigma)_{2} \delta_{2} \\
& \left.-2\left(1-\alpha^{2}\right) \delta_{3}(\delta+\sigma)_{3}\right] \\
& +\frac{1}{4} A\left[\left(1-\alpha^{2}\right)^{2} w_{3}^{2}-\left(1+\theta^{2}\right)^{2} w_{2}^{2}\right] \exp (2 \sigma-2 \delta, \\
& G_{3}^{3}=-G_{2}^{2}+A\left[\left(1-\alpha^{2}\right)(\delta+\sigma)_{3}\left\{\left(\theta^{2}+\alpha^{2}\right) \beta_{3}-2 \delta_{3}\right\}-\Delta(\delta+\sigma)\right. \\
& \left.-\left(1+\theta^{2}\right)(\delta+\sigma)_{2}\left\{\left(\theta^{2}+\alpha^{2}\right) \beta_{2}+2 \delta_{2}\right\}\right] \text {, } \\
& G_{1}^{0}=\frac{1}{2} A\left[\Delta w+\left(1+\theta^{2}\right) w_{2}(3 \sigma-\delta)_{2}+\left(1-\alpha^{2}\right) w_{3}(3 \sigma-\delta)_{3}\right] \exp (2 \sigma-2 \delta) \text {, } \\
& G_{0}^{1}=\frac{1}{2} A\left[-\Delta w-2 w \Delta(\sigma-\delta)+\left(1+\theta^{2}\right)\left\{2 w\left(\delta_{2}^{2}-\sigma_{2}^{2}\right)-w_{2}(3 \sigma-\delta)_{2}\right\}\right. \\
& \left.+\left(1-\alpha^{2}\right)\left\{2 w\left(\delta_{3}^{2}-\sigma_{3}^{2}\right)-w_{3}(3 \sigma-\delta)_{3}\right\}\right] \\
& -\frac{1}{2} A\left[w^{2}\left\{\Delta w+\left(1+\theta^{2}\right) w_{2}(3 \sigma-\delta)_{2}+\left(1-\alpha^{2}\right) w_{3}(3 \sigma-\delta)_{3}\right\}\right. \\
& \left.+2 w\left\{\left(1+\theta^{2}\right) w_{2}^{2}+2\left(1-\alpha^{2}\right) w_{3}^{2}\right\}\right] \exp (2 \sigma-2 \delta), \\
& G_{3}^{2}=A\left(1+\theta^{2}\right)\left[(\delta+\sigma)_{23}-\left(\beta_{3}+\frac{\alpha}{\theta^{2}+\alpha^{2}}\right)(\delta+\sigma)_{2}-\left(\beta_{2}+\frac{\theta}{\theta^{2}+\alpha^{2}}\right)\right. \\
& \left.\times(\delta+\sigma)_{3}+\delta_{2} \delta_{3}+\sigma_{2} \sigma_{3}-\frac{1}{2} w_{2} w_{3} \exp (2 \sigma-2 \delta)\right], \\
& G_{2}^{3}=\frac{1-\alpha^{2}}{1+\theta^{2}} G_{3}^{2},
\end{aligned}
$$

where

$$
A=\frac{1}{a^{2}\left(\theta^{2}+\alpha^{2}\right)} \exp (-2 \beta),
$$

and

$$
\Delta \beta=\left(1+\theta^{2}\right) \beta_{22}+\left(1-\alpha^{2}\right) \beta_{33}+\theta \beta_{2}-\alpha \beta_{3} .
$$

In the above expressions and in the following, the lower suffixes 2 and 3 after an unknown function denote partial differentiation with respect to $\theta$ and $\alpha$ respectively. 


\section{Solution of the empty space-time field equations}

Einstein's vacuum field equations are

$$
G_{j}^{i}=0 .
$$

A solution of the equation $G_{2}^{2}+G_{3}^{3}=0$ is

$$
\sigma+\delta=k=\text { constant. }
$$

Then the equations corresponding to $G_{3}^{2}=G_{2}^{3}=0$ imply

$$
\begin{aligned}
4 \delta_{2} \delta_{3}-w_{2} w_{3} e^{2 k-4 \delta} & =0, \\
\frac{\partial}{\partial \theta}\left(e^{2 \delta-k}\right) \frac{\partial}{\partial \alpha}\left(e^{2 \delta-k}\right) & =\frac{\partial w}{\partial \theta} \frac{\partial w}{\partial \alpha} .
\end{aligned}
$$

Hence a solution is

$$
w=e^{2 \delta-k}+C,
$$

where $C$ is a negative constant satisfying the relation

$$
-2 e^{2 \delta-k}<C<0 \text {. }
$$

Now use of the results (3.2) and (3.3) simplify the field equation $G_{1}^{0}=0$ to the form

$$
\Delta\left(e^{-2 \delta}\right)=0 \text {. }
$$

This is a linear partial differential equation of second order. By using the method of separation of variables, and the method of Frobenius, the solution of (3.4) is

$$
\begin{aligned}
e^{-2 \delta}=1- & {\left[C_{0}^{\prime}\left(1+\sum_{s=1}^{\infty} \frac{-n^{2}\left(2^{2}-n^{2}\right)\left(4^{2}-n^{2}\right) \ldots\left\{(2 s-2)^{2}-n^{2}\right\}}{(2 s) !} \alpha^{2 s}\right)\right.} \\
& \left.+C_{1}^{\prime} \alpha\left(1+\sum_{s=1}^{\infty} \frac{\left(1^{2}-n^{2}\right)\left(3^{2}-n^{2}\right) \ldots\left\{(2 s-1)^{2}-n^{2}\right\}}{(2 s+1) !} \alpha^{2 s}\right)\right] \\
\times & {\left[D_{0}^{\prime} \theta^{-n}\left(1+n \sum_{s=1}^{\infty} \frac{(-1)^{s}(n+s+1)(n+s+2) \ldots(n+2 s-1)}{s !}\left(\frac{1}{2 \theta}\right)^{2 s}\right)\right.} \\
& \left.+D_{1}^{\prime} \theta^{n}\left(1+n \sum_{s=1}^{\infty} \frac{(-1)^{s+1}(s+1-n)(s+2-n) \ldots(2 s-1-n)}{s !}\left(\frac{1}{2 \theta}\right)^{2 s}\right)\right],
\end{aligned}
$$

where $C_{0}^{\prime}, C_{1}^{\prime}, D_{0}^{\prime}$ and $D_{1}^{\prime}$ are arbitrary constants and $n$ is a constant parameter of the family.

By using equations $(3.2,3.3,3.4)$ it is easy to show that the field equation $G_{0}^{0}=0$ reduces to the form

$$
\Delta \beta=0 .
$$

This is again a linear partial differential equation. The rest of the field equations corresponding to

$$
G_{1}^{1}=G_{2}^{2}=G_{3}^{3}=G_{0}^{1}=0
$$


are satisfied identically. The general solution of equation (3.6) is

$$
\begin{gathered}
\beta=\left[C_{0}\left(1-\sum_{s=1}^{\infty} \frac{m^{2}\left(2^{2}-m^{2}\right)\left(4^{2}-m^{2}\right) \ldots\left\{(2 s-2)^{2}-m^{2}\right\}}{(2 s) !} \alpha^{2 s}\right)\right. \\
\left.\quad+C_{1} \alpha\left(1+\sum_{s=1}^{\infty} \frac{\left(1^{2}-m^{2}\right)\left(3^{2}-m^{2}\right) \ldots\left\{(2 s-1)^{2}-m^{2}\right\}}{(2 s+1) !} \alpha^{2 s}\right)\right] \\
\quad \times\left[D_{0} \theta^{-m}\left(1+m \sum_{s=1}^{\infty} \frac{(-1)^{s}(m+s+1)(m+s+2) \ldots(m+2 s-1)}{s !}\left(\frac{1}{2 \theta}\right)^{2 s}\right)\right. \\
\left.\quad+D_{1} \theta^{m}\left(1+m \sum_{s=1}^{\infty} \frac{(-1)^{s+1}(s+1-m)(s+2-m) \ldots(2 s-1-m)}{s !}\left(\frac{1}{2 \theta}\right)^{2 s}\right)\right],
\end{gathered}
$$

where $C_{0}, C_{1}, D_{0}$ and $D_{1}$ are arbitrary constants and $m$ is a constant parameter of the family.

Now for the metric (2.3) to be the metric of an asymptotically flat space the following conditions are to be satisfied

and

$$
D_{1}^{\prime}=0, \quad n=1
$$

Thus

$$
D_{1}=0, \quad m>0 \text {. }
$$

$$
\begin{aligned}
e^{-2 \delta}=1-\left[C_{0}^{\prime}(1-\right. & \left.\left.\sum_{s=1}^{\infty} \frac{\left(2^{2}-1\right)\left(4^{2}-1\right) \ldots\left\{(2 s-2)^{2}-1\right\}}{(2 s) !} \alpha^{2 s}\right)+C_{1}^{\prime} \alpha\right] \\
& \times\left[\frac{D_{0}^{\prime}}{\theta}\left(1+\sum_{s=1}^{\infty} \frac{(-1)^{8}(s+2)(s+3) \ldots(2 s)}{s !}\left(\frac{1}{2 \theta}\right)^{2 s}\right)\right]
\end{aligned}
$$

and

$$
\begin{aligned}
\beta=\left[C_{0}\left(1-\sum_{s=1}^{\infty} \frac{m^{2}\left(2^{2}-m^{2}\right) \ldots\left\{(2 s-2)^{2}-m^{2}\right\}}{(2 s) !} \alpha^{2 s}\right)\right. \\
\left.+C_{1} \alpha\left(1+\sum_{s=1}^{\infty} \frac{\left(1^{2}-m^{2}\right)\left(3^{2}-m^{2}\right) \ldots\left\{(2 s-1)^{2}-m^{2}\right\}}{(2 s+1) !} \alpha^{2 s}\right)\right] \\
\quad \times\left[D_{0} \theta^{-m}\left(1+m \sum_{s=1}^{\infty} \frac{(-1)^{s}(m+s+1)(m+s+2) \ldots(m+2 s-1)}{s !}\left(\frac{1}{2 \theta}\right)^{2 s}\right)\right]
\end{aligned}
$$

is a class of solutions representing the exterior gravitational field of a rotating stationary oblate spheroid.

The author has studied the structure of this class of solution, and the results will be published elsewhere. It is interesting to note here that according to the Petrov classification, the Riemann curvature tensor of the metric obtained above is of type III. 


\section{Analysis for the interior solution}

It is well known that the gravitational field owes much to the interior distribution of matter. It is very difficult, however, to obtain an exact solution for the interior region of a rotating oblate spheroid. Recently, Roos [9] has proved that an analytic axisymmetric and stationary distribution of dust cannot be a source for the gravitational field described by the Kerr metric. Here we will prove the same result for the more general axisymmetric stationary metric (2.3).

The energy momentum tensor for an incoherent dust distribution is

$$
T^{i j}=\varepsilon u^{i} u^{j},
$$

where $\varepsilon$ is the energy density of the dust. To write out the field equations explicitly we need expressions for the components of the four-velocity vector $u^{i}$. With the definitions

$$
\frac{d \phi}{d t}=\Omega \quad \text { and } \quad V=(\Omega-w) \exp (\sigma-\delta),
$$

we readily find that the contravariant and the covariant components of the fourvelocity $u^{i}$ are given by

$$
u^{0}=\frac{\exp (-\delta)}{\sqrt{\left[\left(1-V^{2}\right)\right]}}, \quad u^{1}=\Omega u^{0}, \quad u^{2}=u^{3}=0
$$

and

$$
u_{0}=-\frac{\exp \delta}{\sqrt{\left(1-V^{2}\right)}}\{1+w V \exp (\sigma-\delta)\}, \quad u_{1}=\frac{V}{\sqrt{\left(1-V^{2}\right)}} \exp \sigma, \quad u_{2}=u_{3}=0 .
$$

Hence the components of the energy momentum tensor of an incoherent dust are

$$
\begin{aligned}
T_{0}^{0} & =\frac{1}{\Omega} T_{0}^{1}=-\frac{\varepsilon[1+w V \exp (\sigma-\delta)]}{1-V^{2}}, \\
T_{1}^{0} & =\frac{1}{\Omega} T_{1}^{1}=\frac{\varepsilon V}{1-V^{2}} \exp (\sigma-\delta), \\
T_{\mu}^{i} & =T_{i}^{\mu}=0 \quad(\mu=2,3 \text { and } i=0,1,2,3) .
\end{aligned}
$$

Einstein's field equations for the interior region are

$$
G_{j}^{i}=8 \pi T_{j}^{i} \text {. }
$$

Hence from the results (2.7), (2.10), (4.7) and (4.8), it is easy to show that the solutions

$$
\begin{aligned}
\sigma+\delta & =k, \\
w & =C+\exp (2 \delta-k),
\end{aligned}
$$


hold for the interior region also. Now the equations corresponding to $G_{2}^{2}=G_{3}^{3}=0$ imply the result $\Delta \beta=0$ and, from (2.8) and (2.9), it is easy to show that

$$
C^{2} G_{1}^{0}+G_{0}^{1}=0 .
$$

Equation (4.8) also yields the result

$$
C^{2} T_{1}^{0}+T_{0}^{1}=0 .
$$

By using equations $(4.5,4.6)$ and simplifying the result so obtained we get the equation

$$
(\Omega-C)^{2} \exp (2 \delta-k)=-C(\Omega-C)^{2}
$$

which implies that either

$$
\Omega=C
$$

or

$$
\exp (2 \delta-k)=-C .
$$

If (4.11) is true then $V=1$ and hence $T_{0}^{0}$ and $T_{1}^{1}$ are infinite. This is not physically acceptable because it gives an infinite density for the dust distribution. In the case (4.12) then $w=0$. The space-time metric is not stationary but static. This proves the following proposition.

Proposition. An analytic axisymmetric and stationary distribution of dust cannot be a source for the gravitational field described by the metric (2.3).

\section{Conclusion}

The class of solutions of the vacuum field equations, discussed in Section 3 are free from singularity for $\theta>\frac{1}{2}$. They have singularities for $\theta<\frac{1}{2}$, hence the spheroid $\theta=$ constant $>\frac{1}{2}$ may be a source of the vacuum field solutions.

The non-existence of stationary rotating dust sources for the rotating axisymmetric space-time metric is physically reasonable because without pressure there is no force to counterbalance the gravitation in directions parallel to the axis of rotation. The rotation could stabilize a dust cloud at best in each surface which is parallel to the equatorial plane.

\section{Acknowledgement}

The author's grateful thanks are due to Professor J. K. Rao for his valuable guidance and for suggesting improvements in the paper. 


\section{References}

[1] W. B. Bonnor, J. Math. Mech. 6 (1957), 203.

[2] S. Chandrasekhar and J. L. Friedman, Ap. J. 175 (1972), 379.

[3] R. P. Kerr, Phys. Rev. Lett. 11 (1963), 237.

[4] L. Marder, Proc. Roy. Soc. London A 244 (1958), 524.

[5] M. Misra, Proc. Nat. Inst. Sci. India A 26 (1960), 675.

[6] A. Papapetrou, Ann. Physik VI F 12 (1953), 309.

[7] L. K. Patel, J. Austral. Math. Soc. B 19 (1975), 140.

[8] N. Rosen, Bull. Res. Council Israel 3 (1954), 328.

[9] W. Roos, GRG. J. 7 (1976), 431.

[10] J. Weber and J. A. Wheeler, Rev. Mod. Phys. 29 (1957), 509.

Department of Mathematics and Statistics

Sardar Patel University

Vallabh Vidyanagar-388 120

Via. Anand, Gujarat

India 